\title{
Virtual Scleral Lens Fitting over Large Filtering Bleb Using Corneal-Scleral Topography
}

\author{
DeNaeyer G ${ }^{1}$, Sanders DR $^{2^{*}}$ \\ ${ }^{1}$ Optometrist at Arena Eye Surgeons, USA \\ ${ }^{2}$ Center for Clinical Research and President and CEO, Visionary Optics LLC, USA
}

Received: 21 March, 2018; Accepted: 19 April, 2018; Published: 28 April, 2018

*Corresponding author: Sanders DR, Center for Clinical Research and President and CEO, Visionary Optics LLC, 386 N York, Suite 202, Elmhurst, IL 60126, USA, Fax: 540-636-7976; Tel: 630-530-9700; E-mail: drsmd@drsmd.com

\begin{abstract}
Scleral lens fitting can be challenging in the presence of localized conjunctival/scleral elevations (pingueculae or filtering blebs) in the area of the lens landing zone. We report on a case that was virtually fit successfully with a new corneal-scleral topography system. The patient had a history of keratoconus and subsequent penetrating keratoplasty as well as a previous trabeculectomy and glaucoma shunt surgery. Scleral topography revealed a $7 \mathrm{~mm}$ wide and $2 \mathrm{~mm}$ deep supero-temporal elevation. The topography software contained a "cut-out" tool which removed the elevation for the purposes of scleral lens fit since inclusion of that area could adversely affect fit when a notch or a localized vault of the scleral lens is used to avoid this area. Customized fitting software recommended a base curve (BC) of $42 \mathrm{D}$ and $288 \mu$ of posterior haptic toricity to achieve $300 \mu$ of central corneal clearance and haptic alignment. The diameter and elevation of the bleb from scleral topography were used to order a notched lens and one with a customized back surface haptic. Both lenses were successfully fit in the same eye, equally comfortable with improved vision. No fitting complications resulted from either design and both lenses were well stabilized with posterior haptic toricity. The patient currently wears the lens with the customized back surface haptic.
\end{abstract}

Key Words: scleral lenses; scleral obstacle; scleral shape;

\section{Introduction}

Localized conjunctival/scleral elevations in the area of the lens landing zone especially after glaucoma surgery (trabeculectomy and/or glaucoma drainage device placement) represent unique challenges in the fitting of scleral lenses. The relatively large diameter of a scleral contact lens will result in haptic overlap of any paralimbal conjunctival bleb. The firm nature of gas-permeable material doesn't allow it to drape over potential obstacles, not only resulting in a fitting mismatch but the potential for the scleral lens to cause trauma to the elevated conjunctival tissue. Therefore advanced fitting designs are necessary to bypass conjunctival blebs to achieve safe and successful wear. One common design technique is to fit a scleral lens that has a notched haptic of approximately the same size as the conjunctival bleb [1]. Another approach is to create a lifted section of the haptic that will allow it to vault over the elevated conjunctiva. This case demonstrates the value of corneal-scleral topography in the design of scleral lenses incorporating both design techniques.

\section{Case Report}

The patient was a 64-year-old woman with keratoconus who had previous corneal transplant and cataract surgery of her right eye. A traumatic accident resulted in aphakia and need for repeat penetrating keratoplasty. She subsequently developed glaucoma, which was in part managed with a trabeculectomy. She had an additional shunt procedure secondary to failure of the previous filtering bleb (Figure 1). Her best spectacle correction was

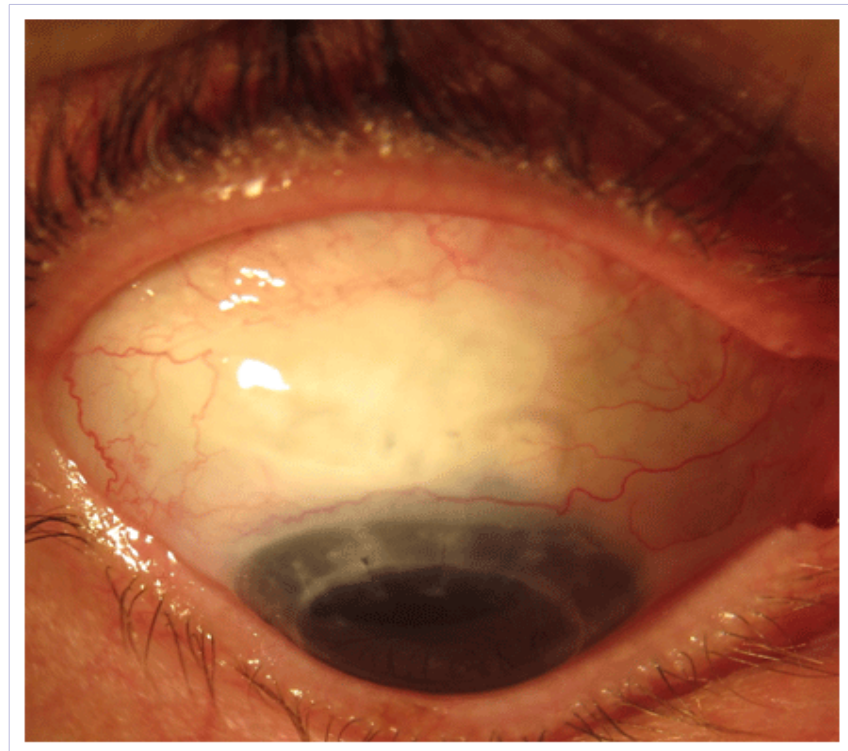

Figure 1: Clinical appearance of the eye demonstrating the previous corneal transplant and the supero-temporal filtering bleb

count fingers vision with $a+4.00-4.00 \times 072$. Prior to scleral lens fitting, the patient was measured with the sMap3D Cornealscleral topographer (Precision Ocular Metrology, Los Angeles, CA) [2]. The sMap3D is a fluorescence-based structured light topographer that allows for the simultaneous mapping of corneal and scleral topography (elevation). The major components of the instrument are a single digital light processing (DLP) projector and two cameras that are imaging the eye at two different angles. During the measurement, the DLP projector illuminates the eye with a sequence of green and blue frames. The green 
illumination is uniform and is used for imaging the features on the eye such as the blood vessels. Recording is used before and after the imaging process to ensure that the eye does not move during the measurement. The blue illumination consists of alternating patterns of horizontal and vertical lines, which is termed structured light illumination. These patterns are used to excite the fluorescein and simultaneously obtain a fluorescence based measurement from the cornea and the sclera. The pattern sequence is designed so that each portion of the eye surface is illuminated by at least one of the patterns in the sequence. The excited fluorescent patterns are imaged by two cameras, and the angle between the cameras allows for a triangulation measurement. This measurement is performed for each of the patterns in the structured light illumination.
The clinical measurement is performed as follows: a drop of fluorescein sodium $0.25 \%$ and benoxinate hydrochloride $0.4 \%$ ophthalmic solution (Bausch and Lomb, Tampa, FL.)was placed in the eye, the upper and lower lids were retracted, and the patient was instructed to look at the fixation light in the straight-gaze, and, after focusing in the corneal center, the button on the slit lamp joystick was depressed to take the first exam. Again with lid retraction the patient was then instructed to look at the fixation light which was now in down-gaze and after focusing in the superior limbus, the button on the joystick was depressed. The process was repeated again with the fixation light now in up-gaze, the image centered on inferior limbus and the examiner retracting both lids (Figure 2, top). The images were stitched together into a single topographic image using a proprietary algorithm (Figure 2, bottom).
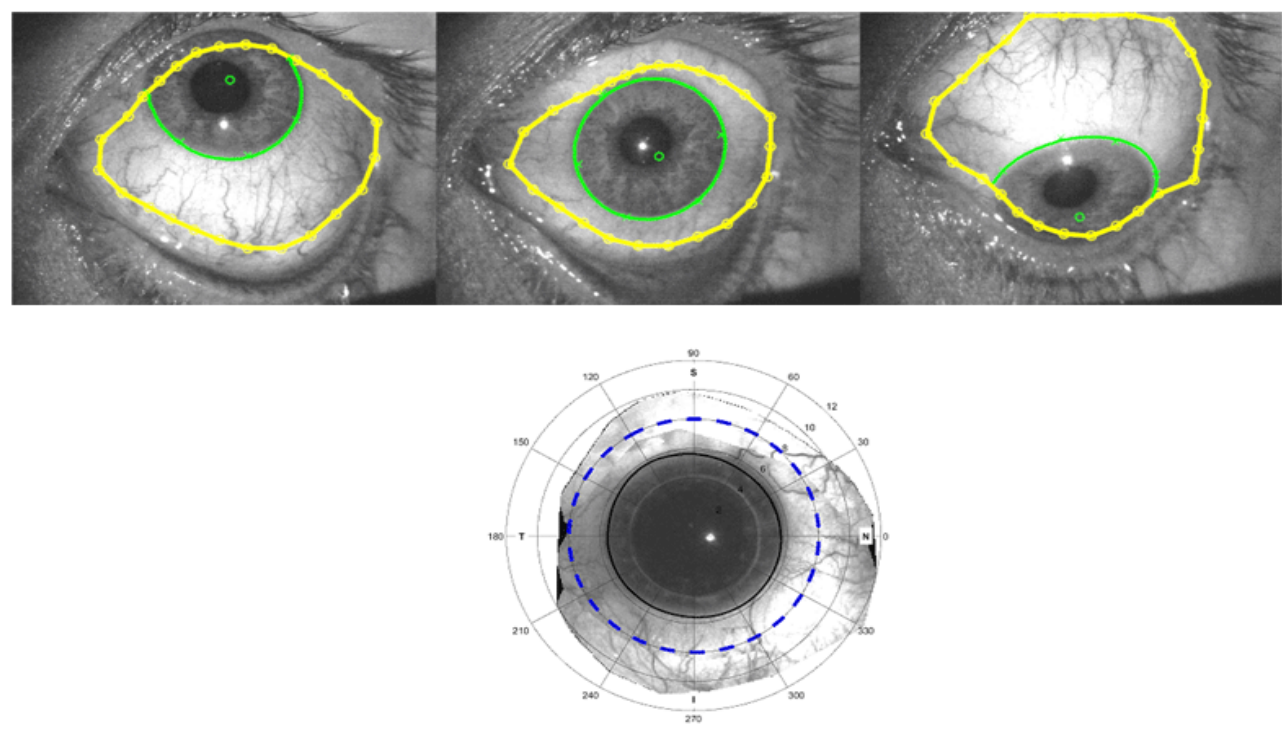

Figure 2: The up, straight and down-gaze images (top) are obtained. Yellow lines demarcate the measurable conjunctival/scleral surface and the green lines demarcate the limbus. The image on the bottom demonstrates the extent of ocular surface coverage obtained by stitching these 3 images above on a background of standard polar axis coordinates. The blue dotted line represents a 16-mm diameter through the center of the cornea and the black circle represents the limbus

The 3D image map (Figure 3) demonstrated the elevated area of the bleb superiorly when compared to the surface inferiorly. The scleral elevation map (Figure 4A) further identified the superotemporal filtering bleb, the size of which was quantitated using the measurement ruler tool. The bleb was approximately $7 \mathrm{~mm}$ wide and $2 \mathrm{~mm}$ deep (assuming out to a diameter of $15.5 \mathrm{~mm}$ ). The "cut out" tool, which removed the elevation area, was utilized (Figure 4B) since inclusion of that area would adversely affect the fit when a notch or a localized vault of the scleral lens was used to avoid this area. The virtual fit without removing the bleb area recommended a base curve (BC) of approximately 34D in order to achieve $300 \mu$ of central clearance while it recommended a BC of 42D with the cut out tool. The predicted posterior haptic toricity without removing the bleb area was $539 \mu$ and using the cutout tool was $288 \mu$. Figure $5 \mathrm{~A}$ demonstrates the simulated fluorescein pattern showing edge lift with fluorescein predicted to leak from the edge of the lens superonasally if the lens is fit

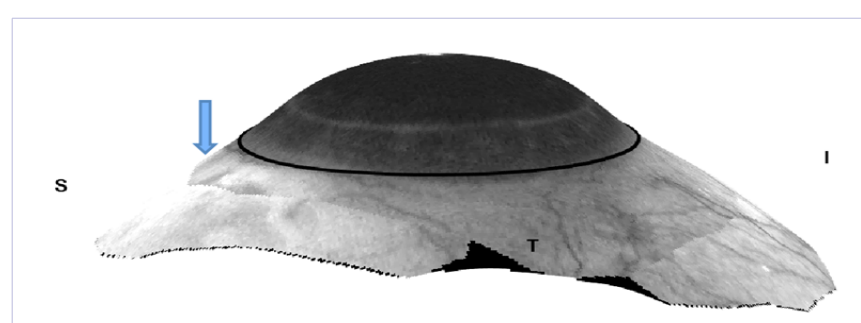

Figure 3: Three dimensional image map demonstrating the elevated filtering bleb superiorly (arrow).

without accounting for the bleb elevation; figure $5 \mathrm{~B}$ shows no such leakage when the area of the bleb is "cut out".

The BC and posterior haptic toricity values with the use of the cut out tool as well as the size of the bleb as measured in Figure $4 \mathrm{~A}(7 \mathrm{~mm} \times 2 \mathrm{~mm})$ were used to order a $15.5 \mathrm{~mm}$ notched lens for this eye (Figure 6). The $\mathrm{BC}$ and the quantitative information 

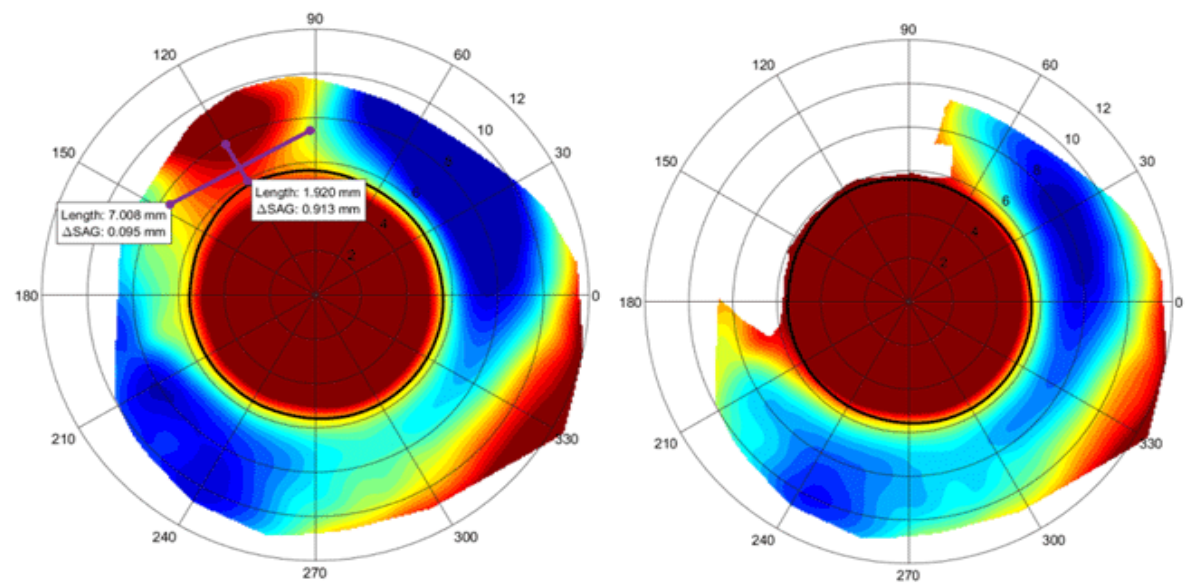

Figure 4: Scleral elevation maps of the eye. Elevated areas are demonstrated by the red and yellow colors while areas of surface depression are in blue. (A) The ruler tool of the sMap3D software quantitates the width and the depth of the elevated filtering bleb out to a $15.5 \mathrm{~mm}$ diameter. (B) The cutout tool outlines the area of the filtering bleb and removes it from consideration in determining the clearances and peripheral fit of the remainder of the lens
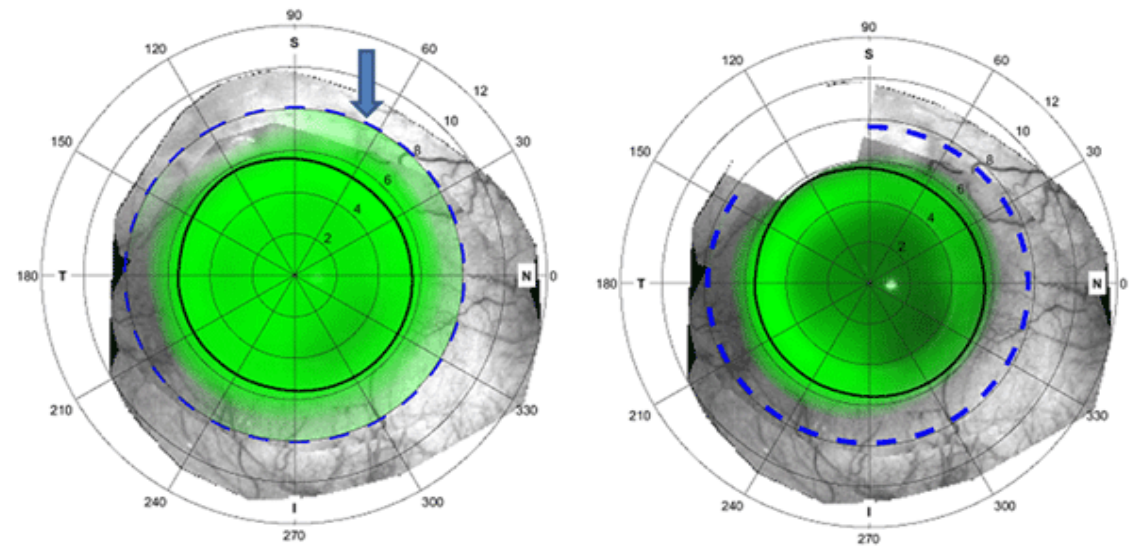

Figure 5: Simulated fluorescein pattern of the eye after fluorescein placed in the bowl of the scleral lens. The blue circle outlines the edge of the lens. (A) without cutting out or accounting for the filtering bleb elevation. Note the area of edge lift at the edge of the lens superonasally (arrow). (B) Use of a cut out to better fit the eye when a notch is used. No edge lift is present

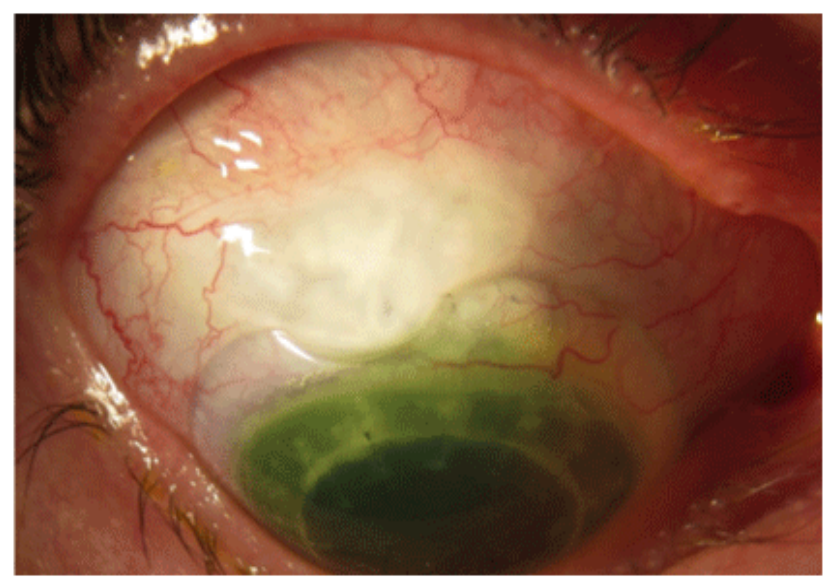

obtained directly from the scleral shape plots (Figure 7) at $13 \mathrm{~mm}, 14 \mathrm{~mm}$ and $15.5 \mathrm{~mm}$ diameters were input directly into the manufacturing lathe to produce a custom peripheral haptic scleral lens for the same eye (Figures 8,9).

Both the notched and custom peripheral haptic scleral lenses were successfully fit on the same eye of this patient and were equally comfortable with improved vision to $20 / 40$. No fitting complications resulted from either design; neither required design modifications and both lenses were well stabilized with posterior haptic toricity. The patient currently wears the custom peripheral haptic scleral lens.

Figure 6: Appearance of the notched lens on the eye 

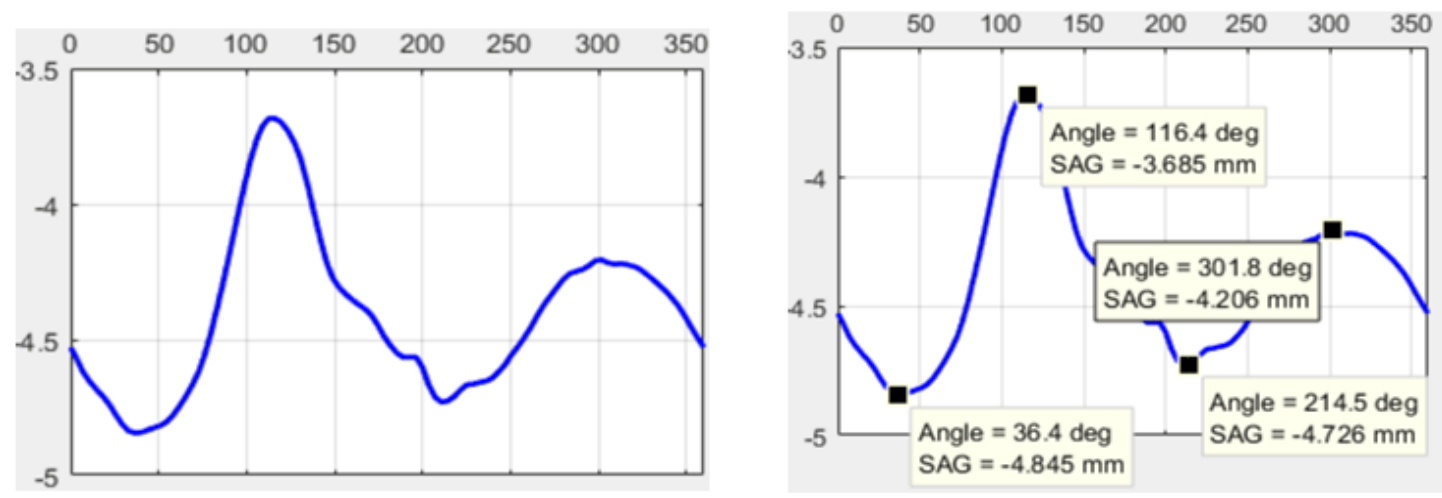

Figure 7: Scleral shape plots. (A) Qualitative plot. (B) Quantitative data obtained from the sMap3D scleral topographer was input into the manufacturing lathe software to produce the scleral lens shown in (Figures 8 and 9)
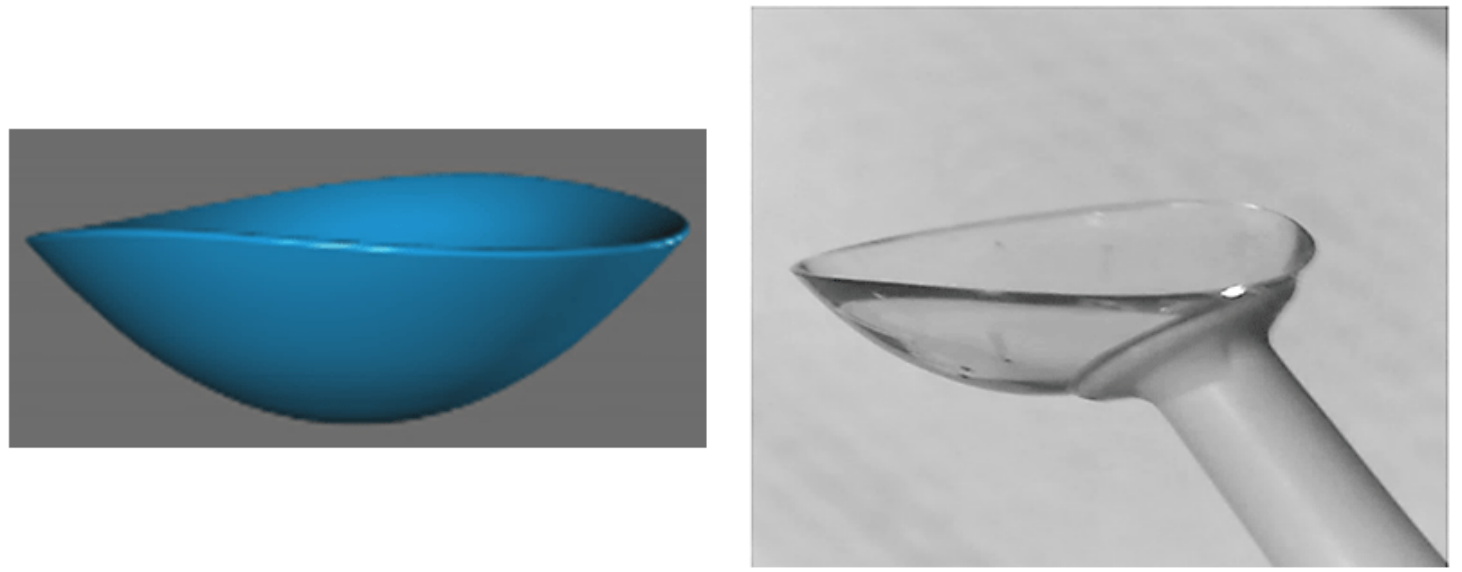

Figure 8: Customized peripheral back surface scleral lens designed with the parameters obtained by the sMap3D wide field Corneal-scleral topographer. (A) Computer Aided Design (CAD) image from manufacturing software. (B) Actual manufactured lens

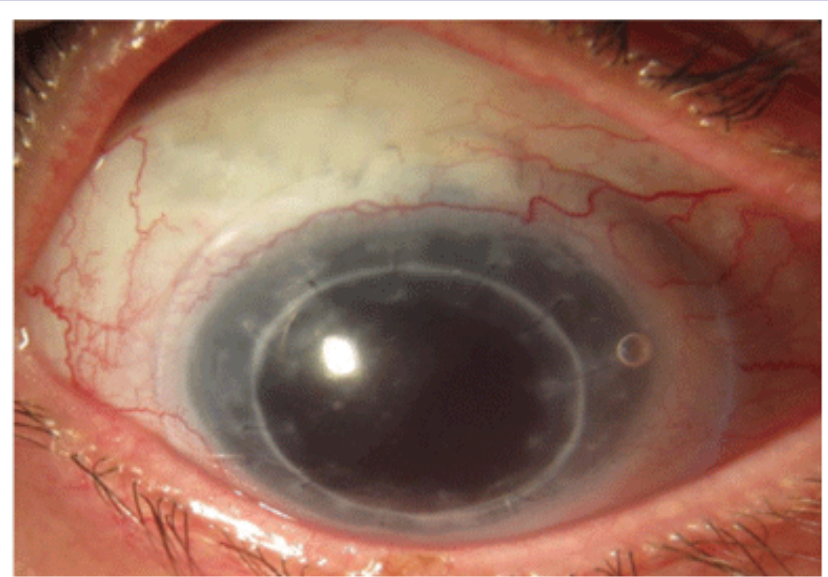

Figure 9: Appearance of the customized peripheral back surface scleral lens on the eye

\section{Discussion}

The only published articles reporting scleral lenses in cases with filtering blebs in the presence of a glaucoma drainage device involved the use of the PROSE lens [4,5]. In the first report [4], in
2010, 5 cases were reported as having been fit however 1 case $(20 \%)$ had erosion of the conjunctiva over the device requiring surgical revision and the patient was advised against further use of the scleral lens. The second report [5], in 2016, reported on 7 eyes. In 2 eyes (29\%), the lenses could not be fit at all because of "scleral/conjunctival irregularities" and an additional 3 cases (43\%) were reported as "fit achieved but not physiologic". This included 1 case with intermittent epithelial erosion and conjunctival irritation. In the remaining 2 cases (29\%), fit was reported as achieved with good physiologic outcome however in both of these cases the standard glaucoma procedure had to be modified in anticipation of the use of the PROSE lenses. These reports reinforce the difficulties that such cases can bring to the scleral lens fitting process

Eliminating lens interaction for the patient who has a paralimbal conjunctival bleb and needs a gas-permeable (GP) lens to mask irregularity is critical to avoid mechanical contact lens related injury. Utilizing a corneal gas-permeable lens design can be an option if the lens properly centers. However, for patients with highly irregular corneas, a well-fit, centered corneal GP is often not possible. The increased diameter of a scleral lens often 
improves stability but the consequence is that the haptic of the lens has the potential to overlap the area of conjunctival elevation. Designing scleral lenses with a notch or localized elevation to bypass this area can be extremely challenging and require multiple remakes when fitting with diagnostic lenses. Accurate assessment for lens design concerning corneal clearance and haptic alignment can't begin until a lens is manufactured that bypasses the conjunctival bleb. Measurement using cornealscleral topography eliminates the guess work. The "cut out" tool allows the fitter to properly design a scleral lens that vaults the cornea by a predetermined amount and a haptic design that matches the sclera surface. The recommended lens parameters without elimination of the elevation highlights the difficulties that practitioners deal with when they have to rely on diagnostic lenses without scleral measurement and fitting software. For this patient; two different lens designs, one with a notch and the other with a custom peripheral haptic, were both successfully fit in the same eye on the first attempt for each.

The use of corneal-scleral topography data dramatically simplified the fitting process making it possible to efficiently fit this eye with both cornea and scleral shape abnormalities using 2 different fitting techniques; notching and customized peripheral haptic fit.

\section{References}

1. DeNaeyer G. Contact Lenses after Trabeculectomy. Contact Lens Spectrum. 2010.

2. DeNaeyer G, Sanders DR, Farajian TS. Surface coverage with single vs. multiple gaze surface topography to fit scleral lenses. Cont Lens Anterior Eye. 2017;40(3):162-169. doi: 10.1016/j.clae.2017.03.009

3. DeNaeyer G, Sanders D, van der Worp E, Jedlicka J, Michaud L, Morrison S. Qualitative assessment of scleral shape patterns using a new wide field ocular surface elevation topographer. J Cont Lens Res Sci. 2017;1(1):12-22.

4. Tanhehco T, Jacobs DS. Technological advances shaping scleral lenses: the Boston ocular surface prosthesis in patients with glaucoma tubes and trabeculectomies. Semin Ophthalmol. 2010;25(5-6):233-238. doi: 10.3109/08820538.2010.518873

5. Nguyen AH, Dastiridou AI, Chiu GB, Francis BA, Lee OL, Chopra V. Glaucoma surgical considerations for PROSE lens use in patients with ocular surface disease. Cont Lens Anterior Eye. 2016;39(4):257-261. 